\title{
Regressor time-shifting to identify longitudinal stability and control derivatives of the Jetstream 3102
}

\section{Sezsy Yusuf, Mudassir Lone, Alastair Cooke and Nicholas Lawson *}

\begin{abstract}
The Jetstream 31 G-NFLA aircraft is used as a national flying laboratory test vehicle for flight dynamics research and teaching purposes. It has been the subject of much theoretical and experimental modelling and therefore, the need for generating validation data through flight testing is critical. In this paper, the aircraft's short period pitch oscillation mode characteristics are identified using data from sixteen flight tests. An identification procedure based on the least squares method and reduced order state-space model is used and the need for pre-processing regressors due to the effects of sensor location and instrumentation delays is highlighted. It has been shown that time-shifting the regressors based on relative locations of the angle of attack vanes and the inertial measurement unit results in significant reductions in uncertainty bounds of the estimated aeroderivatives and also a model that provides a closer match to flight test data. The estimated models are validated using separate flight test data and the variations in aeroderivatives over a range of airspeeds and centre of gravity positions are also presented.
\end{abstract}

Keywords : Parameter estimation, short period pitch oscillation, regressor corrections

\section{Nomenclature}

$\begin{array}{llll}c & \text { Mean aerodynamic chord [m] } & \alpha & \text { Angle of attack [deg] } \\ C_{L} & \text { Lift coefficient } & \alpha_{m} & \text { Measured angle of attack [deg] } \\ C_{M} & \text { Pitching moment coefficient } & \alpha_{c} & \text { Corrected angle of attack [deg] } \\ I_{y y} & \text { Aircraft pitch inertia }\left[\mathrm{kgm}^{2}\right] & \eta & \text { Elevator deflection angle [deg] } \\ p, q, r & \text { Angular rates about the } x, y, z \text { axes [deg/s] } & \theta & \text { Parameter vector } \\ q_{m} & \text { Measured pitch rate }[\mathrm{deg} / \mathrm{s}] & \hat{\theta} & \text { Identified parameter vector } \\ q_{c} & \text { Corrected pitch rate }[\mathrm{deg} / \mathrm{s}] & \sigma & \text { Parameter variance } \\ S & \text { Reference area }\left[\mathrm{m}^{2}\right] & \rho & \left.\text { Air density [kg/m }{ }^{3}\right] \\ t & \text { Time [s] } & \nu & \text { Residual vector } \\ V_{0} & \text { Airspeed at test condition }[\mathrm{m} / \mathrm{s}] & \tau & \text { Inserted time shift } \\ \mathbf{X} & \text { Regressor matrix } & & \\ y & \text { Model output } & & \\ z & \text { Measured output } & & \end{array}$

\footnotetext{
${ }^{*}$ Centre for Aeronautics, School of Aerospace, Transport \& Manufacturing, Cranfield University, United Kingdom 


\section{Introduction}

The impact of time delays and signal noise in aircraft parameter estimation has been studied extensively and numerous methods, summarised in ? and ?, have been proposed to address problems associated with sensor properties such as sampling rates and noise. In this paper, the authors present a case study that demonstrates a practical application of one such method for the identification of short period pitch oscillation (SPPO) mode of the Jetstream 31 G-NFLA aircraft. Results from sixteen flights have been analysed and used to identify key longitudinal aeroderivatives that contribute to the SPPO dynamics and demonstrate the effectiveness of the adopted method, which relies on the time shifting of key regressors to account for factors such as (1) differences in filtering and sampling rates of various onboard sensors, (2) pitch acceleration effects due to non-collocated centre of gravity (CG) and inertial measurement unit (IMU) and, (3) the lag in pitch rate relative angle of attack measurement due to different sensor positions. A secondary aim of this paper is to release data for the Jetstream 31 G-NFLA aircraft (Figure 1) which is used as a flying laboratory by around twenty universities to teach flight dynamics.

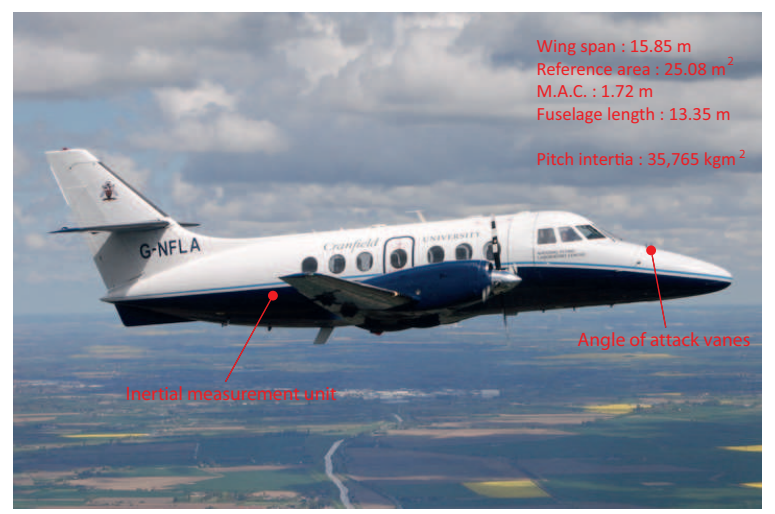

Fig. 1. Cranfield's Jetstream 31 G-NFLA and reference data

To date, the SPPO characteristics of the aircraft have been analysed by students in terms of the modal frequency and damping using classical inspection and logarithmic decrement method. This approach does not provide insight into the aeroderivatives which are the key drivers of the modal frequency and damping. The method detailed in this paper aims to quantify these derivatives through the application of an equation-error based parameter estimation technique to the collected flight test data and hence, provide a reference for future development and analysis of flight dynamic models of this specific aircraft. The aircraft key geometric data are presented in Figure 1.

The paper is structured to follow the classical approach to aircraft system identification. Section 2 details the aircraft model being used and presents the aeroderivatives of interest. This is followed by a brief outline of the time domain least squares approach adopted for parameter estimation in Section 3. A detailed presentation and discussion of data processing and analysis carried out prior to parameter estimation is given in Section 4. Results from the parameter estimation process and the validation of model parameters are given in Section 5. Section 6 provides the reader with a summary of the key discussion points.

\section{Flight dynamic model}

Details of the flight physics that lead to the SPPO mode can be found in numerous text books, such as those written by ? and ?. This mode consists of longitudinal rotation about the CG accompanied by a small amount of plunge motion, resulting in an oscillation in aircraft angle of attack $(\alpha)$ and pitch rate $(q)$. For demonstration purposes it is typically excited through a pulse input to the elevator. The mode is of specific interest due to its relevance to aircraft stability and control as highlighted by certification requirements such as?. 
The following reduced order state-space model approximating the SPPO dynamics was adopted from ?:

$$
\left[\begin{array}{c}
\dot{q} \\
\dot{\alpha}
\end{array}\right]=\left[\begin{array}{cc}
m_{q} & m_{w} \\
1 & \frac{z_{w}}{V_{0}}
\end{array}\right]\left[\begin{array}{c}
q \\
\alpha
\end{array}\right]+\left[\begin{array}{c}
m_{\eta} \\
\frac{z_{\eta}}{V_{0}}
\end{array}\right][\eta]
$$

where pitching moment components are defined as:

$$
\begin{aligned}
m_{q} & =\frac{\rho V_{0}^{2} S c}{2 I_{y y}} \frac{c}{2 V_{0}} C_{m_{q}} \\
m_{w} & =\frac{\rho V_{0}^{2} S c}{2 I_{y y}} C_{m_{\alpha}} \\
m_{\eta} & =\frac{\rho V_{0}^{2} S c}{2 I_{y y}} C_{m_{\eta}}
\end{aligned}
$$

and lift components are defined as:

$$
\begin{aligned}
z_{w} & =\frac{\rho V_{0}^{2} S}{2 M} C_{L_{\alpha}} \\
z_{\eta} & =\frac{\rho V_{0}^{2} S}{2 M} C_{L_{\eta}}
\end{aligned}
$$

The symbols $1 / 2 \rho V_{0}^{2}, S, c, M$ and $I_{y y}$ represent dynamic pressure, reference area, mean aerodynamic chord, aircraft mass and pitch inertia respectively. The identification of derivatives such as $C_{m_{\alpha}}, C_{L_{\alpha}}, C_{L_{\eta}}$ and $C_{m_{\eta}}$ is of specific interest here because these not only provide an indication of longitudinal static stability and control effectiveness, but also allow the validation of data from wind tunnel tests carried out by ? and computational aerodynamic modelling activities done by ??.

It should be noted that no model structure determination process was carried out due to (1) the simplicity of the adopted model and, (2) the classical elevator input used to excite the SPPO mode (tuned by the pilots over numerous flight tests). The assumption that the aircraft's pitch inertia is constant is the most significant at this stage. It has been assumed to be $35,765 \mathrm{kgm}^{2}$ based on work carried out by ?.

\section{Parameter estimation approach}

The adoption of a two state linear model and the decision to address issues with sensor noise and errors by correcting measurements before parameter estimation meant that the aeroderivatives could be obtained through a simple ordinary least squares (OLS) approach. The OLS approach requires the formulation of the following model equation:

$$
y=\mathbf{X} \theta
$$

and the following measurement equation:

$$
z=\mathbf{X} \theta+\nu
$$

where $z \in \mathbb{R}^{N \times 1}, \theta \in \mathbb{R}^{n_{p} \times 1}, \mathbf{X} \in \mathbb{R}^{N \times n_{p}}$ and $\nu \in \mathbb{R}^{N \times 1}$. The parameter vector $\theta$ is obtained by minimising the following cost function:

$$
J(\theta)=\frac{1}{2}[z-\mathbf{X} \theta][z-\mathbf{X} \theta]
$$

such that

$$
\hat{\theta}=\left(\mathbf{X}^{T} \mathbf{X}\right)^{-1} \mathbf{X} z
$$


and therefore under the assumptions of zero mean Gaussian noise, the estimate covariance matrix can be calculated as follows:

$$
\mathrm{E}[(\hat{\theta}-\theta)(\hat{\theta}-\theta)]=\sigma^{2}\left(\mathbf{X}^{T} \mathbf{X}\right)^{-1}
$$

such that $\hat{\theta}$ and the real parameter value $\theta$ lies within $\hat{\theta} \pm 2 \sigma \sqrt{\left(\mathbf{X}^{T} \mathbf{X}\right)^{-1}}$.

In this case the regressor matrices contain $\alpha, q$ and $\eta$ for identifying pitching moment dynamics and only $\alpha$ and $\eta$ for identifying derivatives related to lift, such that the following equations are the model equations:

$$
\begin{aligned}
\dot{q} & =m_{q} q+m_{\alpha} \alpha+m_{\eta} \eta \\
\dot{\alpha}-q & =\frac{z_{w}}{V_{0}} \alpha+\frac{z_{\eta}}{V_{0}} \eta
\end{aligned}
$$

Here $\dot{\alpha}$ and $\dot{q}$ are the dependent variables obtained by differentiating the sensor measurements. The differentiation of raw IMU and alpha vane measurements would lead to significant noise amplification. Therefore, a local smoothing approach was applied to the dependent variables. The smoothing is based on a second order polynomial model that assumes local data points lie on a parabola. Details of this approach can be found in work done by ?

The data collected onboard the aircraft from various sensors at different sampling frequencies are pre-filtered and logged onboard. The IMU data is acquired at $64 \mathrm{~Hz}$ and filtered using a low pass second order Butterworth filter with a cut-off frequency of $8 \mathrm{~Hz}$. The angle of attack vane is sampled at $400 \mathrm{~Hz}$ but an 8 sample average is taken so that angle of attack is actually logged at $50 \mathrm{~Hz}$. Pitch attitude and normal acceleration are measured at $64 \mathrm{~Hz}$ and filtered using a first order low pass filter. Given the fact that all sensor measurements are logged at $50 \mathrm{~Hz}$, the IMU data is acquired with a 1.28 data point advance. For an alpha range of 0 to 15 degrees with zero sideslip and zero bank angle, the current calibration of the angle of attack vanes is accurate to within approx 0.8 degrees based on work carried out by ?. Moreover, the angular rates are obtained with an accuracy of $\pm 0.1 \mathrm{deg} / \mathrm{s}$ from the laser gyros within the IMU as given in the Linton LTN-90 inertial reference system (IRS) specification document.

Data obtained from the IMU is subject to error from two sources: transducer accuracy and discretisation (resolution) error. The specification document for the Linton LTN-90 IRS states that the measured pitch rate has an accuracy of 0.1 $\mathrm{deg} / \mathrm{s}$ and a resolution of $0.0039 \mathrm{deg} / \mathrm{sec}$. As the analysis methodology is based on a time history it can be considered to use 'relative' pitch rate. If the IMU accuracies are assumed to be normally distributed and independent, then the change in pitch rate during any given test could be biased by a maximum amount equal to the transducer error. This bias has been ignored as it will cancel out when computing the change in pitch rate with time. Variations in this bias across a set of tests will partly contribute to the uncertainty in the estimates of the aeroderivatives. Although the resolution error does introduce uncertainty within a single test, as it applies to every data sample, it has also been ignored as a consequence of its small magnitude.

Data from a total of sixteen flight tests were used for parameter estimation, the details of which can be found in Table 1. A further three datasets were isolated for validation purposes. The data covers a range of CG positions ( $24.2 \%$ to $35.7 \%$ ) and airspeeds (142kts to $181 \mathrm{kts}$ equivalent airspeed). These are the ranges normally flown as part of the flight dynamics educational programme at the National Flying Laboratory Centre (NFLC) and have been deemed to have sufficient variations to observe and study trends in the identified aeroderivatives. The SPPO mode is excited using a short pulse input by pulling on the control column and letting go. The SPPO mode is then allowed to develop over a period of approximately five seconds before the pilot inserts a pulse in the opposite sense to bring the aircraft back to it's original pitch attitude.

\section{Regressor time shifting}

The aforementioned signal processing done onboard the aircraft to mitigate noise, along with the knowledge that the angle of attack vane has a degree of lead relative to the IMU measurements due its position towards the nose of the aircraft, led 
Table 1: Flight test matrix

\begin{tabular}{lllllll}
\hline $\begin{array}{l}\text { Flight Test } \\
\text { Number }\end{array}$ & $\begin{array}{l}\text { cg position } \\
{[\% \text { chord }]}\end{array}$ & $\begin{array}{l}\text { Weight } \\
{[\mathrm{kg}]}\end{array}$ & $\begin{array}{l}\text { EAS } \\
{[\mathrm{kts}]}\end{array}$ & $\begin{array}{l}\text { TAS } \\
{[\mathrm{kts}]}\end{array}$ & $\begin{array}{l}\text { Altitude } \\
{[\mathrm{ft}]}\end{array}$ & Note \\
\hline I.A & 28.0 & 6421 & 162 & 178 & 6150 & Identification \\
I.B & 28.3 & 6523 & 158 & 170 & 5720 & Validation \\
I.C & 27.2 & 6425 & 161 & 176 & 6930 & Identification \\
I.D & 28.2 & 6492 & 156 & 177 & 8780 & Identification \\
I.E & 29.7 & 6551 & 160 & 164 & 6210 & Identification \\
II.A1 & 24.2 & 6727 & 161 & 176 & 5030 & Identification \\
II.A2 & 24.2 & 6721 & 142 & 153 & 5020 & Validation \\
II.B1 & 35.7 & 6672 & 163 & 176 & 5070 & Identification \\
II.B2 & 35.7 & 6662 & 181 & 196 & 5200 & Identification \\
II.C1 & 28.1 & 6636 & 150 & 163 & 5040 & Identification \\
II.C2 & 28.1 & 6631 & 162 & 175 & 4950 & Identification \\
II.D1 & 29.8 & 6447 & 161 & 171 & 4190 & Validation \\
II.D2 & 29.8 & 6438 & 169 & 180 & 4390 & Identification \\
II.E1 & 33.3 & 6642 & 179 & 189 & 3500 & Identification \\
II.E2 & 33.3 & 6637 & 161 & 169 & 3450 & Identification \\
II.F2 & 21.8 & 6333 & 163 & 171 & 5200 & Identification \\
\hline
\end{tabular}

to the conclusion that the measured pitch rate $\left(q_{m}\right)$ must be shifted in time to provide more accurate identification of the SPPO dynamics. The time shifting of such measurements has been discussed by ? and ?. The application of time shifting results in the following expression for corrected pitch rate:

$$
q_{c}=q_{m}(t+\tau)
$$

where it is this corrected pitch rate which will be used as a regressor in the parameter estimation algorithm. Furthermore, the $\tau$ time shift parameter can be approximated as follows:

$$
\tau=x_{m} / V
$$

Here, $x_{m}$ is the distance between the angle of attack vane and the IMU. This is effectively the time it takes for an air particle to travel this distance. On the Jetstream 31 G-NFLA, this distance is approximately $7.3 \mathrm{~m}$.

The measured angle of attack also requires correction due to the pitching dynamics of the aircraft being around the CG. By assuming small variations in angle of attack and angular rates, the corrected angle of attack used as a regressor can be calculated as follows:

$$
\alpha_{c}=\alpha_{m}+\frac{q_{c} x_{m}}{V_{T A S}}
$$

The results from the regressor time-shifting process are presented in Figure 2, together with the its effect on identifying the angle of attack dynamics. The corrected angle of attack $\left(\alpha_{c}\right)$, which includes pitch rate effects, allows the model to capture the peak angle of attack observed in flight and avoids the non-minimum phase like feature observed when using the directly measured angle of attack. Note that the accuracy of the sensors for angle of attack and pitch rate are \pm 0.8 deg and $\pm 0.1 \mathrm{deg} / \mathrm{s}$ respectively.

\section{Results and discussion}

The first step in the analysis of the aircraft's SPPO dynamics was to compare the results obtained when using an uncorrected regressor matrix to those obtained when the regressor matrix was corrected as detailed in the previous section. Table 2 presents the results that allow this comparison. The need for using corrected regressors becomes evident when results for the pitch damping derivative $\left(C_{m_{q}}\right)$ are inspected. The direct use of measured pitch rate leads to an unrealistic positive 


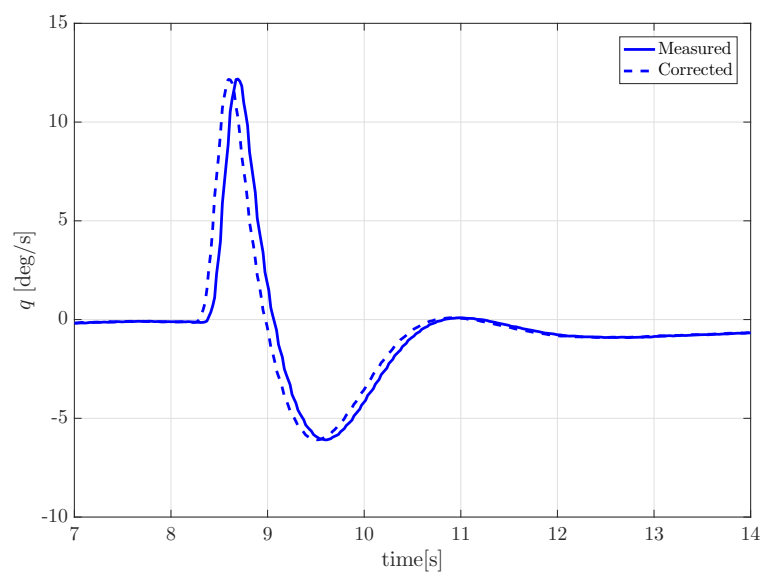

(a) Comparison between $q_{m}$ and $q_{c}$

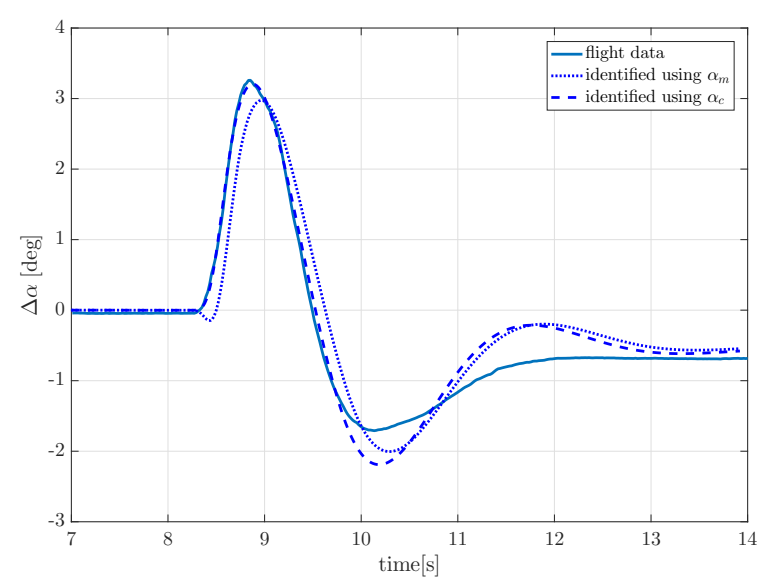

(c) Identification result using measured and corrected $\alpha\left(\alpha_{c}\right.$ and $\left.\alpha_{m}\right)$

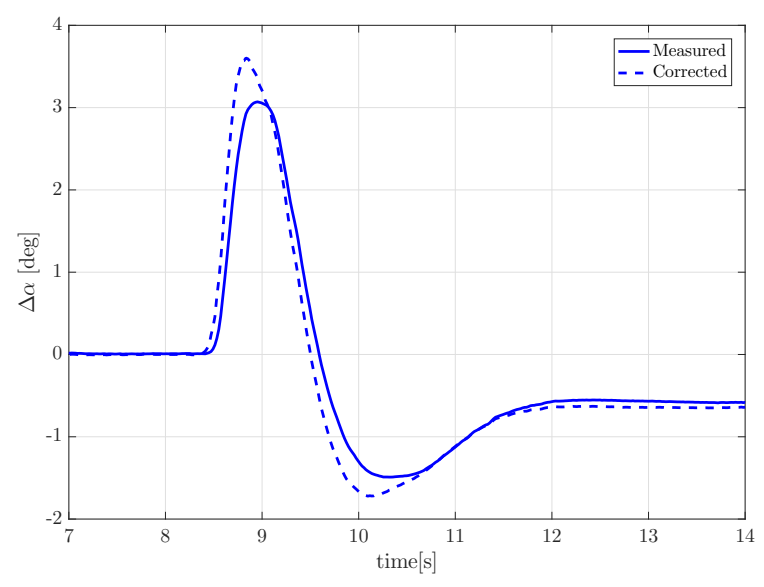

(b) Comparison between $\alpha_{m}$ and $\alpha_{c}$
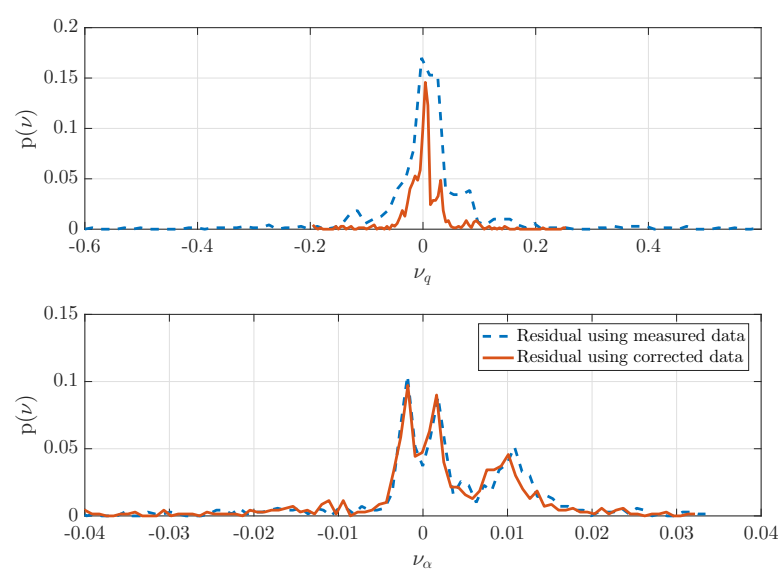

(d) Residual

Fig. 2. Comparison between measurement and corrected $\mathrm{q}$ and $\alpha$ based on sensor location, and the identification results for different $\alpha$ correction. And the residuals from identification result. 
Table 2: Identified parameter and SPPO characteristics using measured and corrected pitch rate

\begin{tabular}{lllllllll} 
& \multicolumn{3}{l}{ Identification using $q_{m}$} & \multicolumn{5}{l}{ Identification using $q_{c}$} \\
Parameter & I.A & \multicolumn{3}{c}{ I.E } & & I.A & I.E & \\
& $\hat{\theta}$ & $\sigma(\%)$ & $\hat{\theta}$ & $\sigma(\%)$ & $\hat{\theta}$ & $\sigma(\%)$ & $\hat{\theta}$ & $\sigma(\%)$ \\
\hline \hline$C_{m_{q}}$ & 8.830 & 32.73 & 9.391 & 24.8 & -15.96 & 8.4 & -19.94 & 4.51 \\
$C_{m_{\alpha}}$ & -1.152 & 6.39 & -1.085 & 5.87 & -0.993 & 2.99 & -0.763 & 2.69 \\
$C_{m_{\eta}}$ & -0.851 & 5.53 & -0.928 & 4.40 & -1.396 & 1.56 & -1.511 & 1.03 \\
$C_{L_{\alpha}}$ & 5.397 & 3.16 & 6.524 & 2.14 & 5.534 & 2.75 & 6.334 & 1.85 \\
$C_{L_{\eta}}$ & -0.168 & 69.09 & -0.3635 & 27.27 & 0.3367 & 32.87 & 0.3091 & 29.27 \\
\hline$\zeta[-]$ & 0.118 & & 0.170 & & 0.352 & & 0.486 & \\
$\omega[\mathrm{rad} / \mathrm{s}]$ & 2.34 & & 2.20 & & 2.38 & & 2.21 & \\
\hline
\end{tabular}

values with relatively large variances. Furthermore, when compared to the estimations done by ? (based on empirical methods), the change in lift coefficient due to elevator deflection $\left(C_{L_{\eta}}\right)$ is underestimated by an order of magnitude and it also has a very high variance. ${ }^{1}$ Given that the parameter estimate variance is being calculated using Equation 11 , such high variances are a direct result of the observed mismatch in the time histories shown in Figure 3. On the other hand, the use of corrected regressors provide realistic results (when compared to past wind tunnel and computational studies) with significantly lower variances in parameter estimates. The time domain SPPO response of the model identified using corrected and measured regressors are presented in Figure 3. It is clear that the use of measured pitch rate and angle of attack leads to an underestimation of the SPPO mode damping. On the other hand, the use of corrected regressors results in a closer approximation of SPPO dynamics both in terms of damping and the maximum observed pitch rates. The results from the application of the regressor corrections and the parameter estimation method for all flights are summarised in Table 3 along with the SPPO mode frequency and damping coefficients.

One of the objectives of this work was to collate a database of aircraft stability and control derivatives for research and educational purposes. The identified aeroderivatives and their variation with respect to true airspeed are presented in Figure 4. The trends observed in the aeroderivatives are as expected for a transport aircraft such as the Jetstream 31 and the reader can compare results with data available in open literature such as ?. The key point here is that the trends align with comments received from test pilots after carrying out longitudinal static stability tests. These tests require the aircraft to be flying with a constant pitch attitude over a range of flight conditions. This in turn means the pilots have to compensate for any atmospheric disturbances while attempting to trim the aircraft. In this case, all the pilots who fly the NFLC Jetstream have reported similar changes in handling qualities through the range of airspeeds tested, with the greatest sensitivity in pitch at the higher tested airspeeds at any given CG position. The observed reduction in $C_{m_{q}}$ and $C_{m_{\eta}}$ with increasing airspeed confirm the statements made by the pilots regarding the needed pitch compensation and difficulties in finding the necessary trim setting. The results obtained for the lift curve slope $\left(C_{L_{\alpha}}\right)$ are also in agreement with past computational fluid dynamic (CFD) simulations and wind tunnel tests.

Data from flights I.B, II.A2 and II.D1 were used for validation where the parameter estimates from the most similar flight conditions were used to simulate the aircraft response. Tolerances suggested by $?$ for SPPO identification have been adopted here: $\pm 1.5 \mathrm{deg}$ in angle of attack and $\pm 2 \mathrm{deg} / \mathrm{s}$ for pitch. Validation results shown in Figures 5, 6 and 7 clearly demonstrate that the identified parameters fit within the tolerances. The slight excursion in pitch rate and minor differences evident for the first validation case are primarily due to the fact that the model's CG position has not been changed and the derivative that captures the effect of wing downwash on the tailplane, i.e. pitching moment due to rate of change in angle of attack has not been explicitly modelled.

\footnotetext{
${ }^{1}$ The empirical method used by $?$ predicts $C_{L_{\eta}}$ to be approximately $2.3 \mathrm{rad}^{-} 1$ at Mach 0.15 .
} 

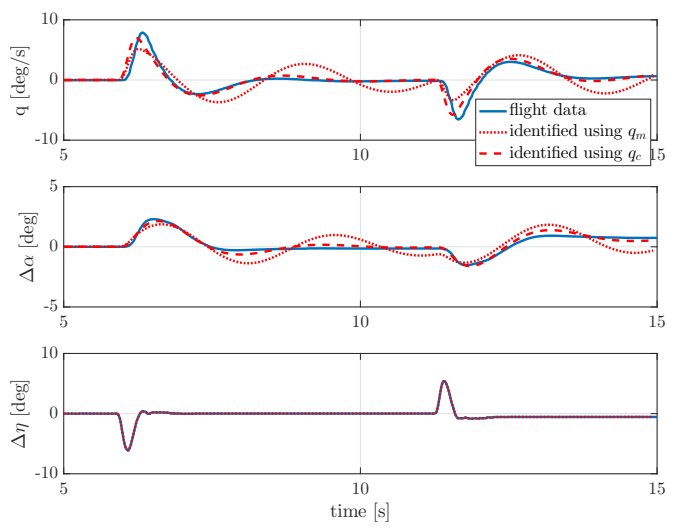

(a) Flight test point I.A
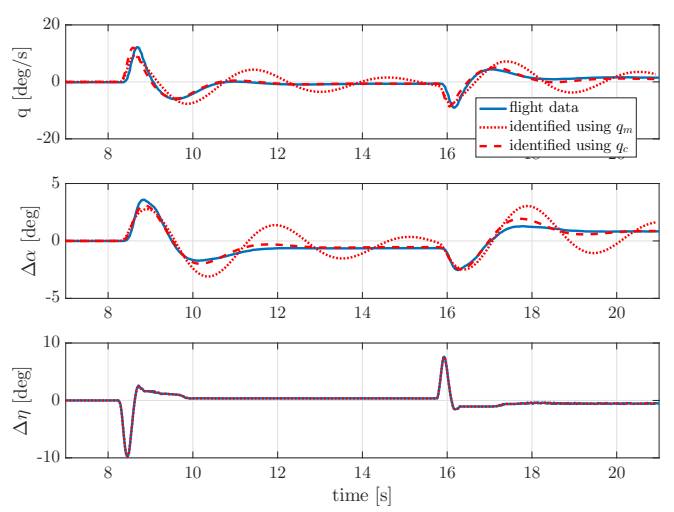

(b) Flight test point I.E

Fig. 3. Flight test and identification result based on corrected pitch rate

Table 3: SPPO characteristics based on identification procedures using corrected $\alpha$ and $q$

\begin{tabular}{|c|c|c|c|c|c|c|c|c|c|c|c|c|}
\hline $\begin{array}{l}\text { Flight Test } \\
\text { Number }\end{array}$ & $\begin{array}{l}C_{m_{q}} \\
\hat{\theta}\end{array}$ & $\sigma(\%)$ & $\begin{array}{l}C_{m_{\alpha}} \\
\hat{\theta}\end{array}$ & $\sigma(\%)$ & $\begin{array}{l}C_{m_{\eta}} \\
\hat{\theta}\end{array}$ & $\sigma(\%)$ & $\begin{array}{l}C_{L_{\alpha}} \\
\hat{\theta}\end{array}$ & $\sigma(\%)$ & $\begin{array}{l}C_{L_{\eta}} \\
\hat{\theta}\end{array}$ & $\sigma(\%)$ & $\begin{array}{l}\omega \\
{[\mathrm{rad} / \mathrm{s}]}\end{array}$ & $\begin{array}{l}\zeta \\
{[-]}\end{array}$ \\
\hline I.A & -15.96 & 8.4 & -0.993 & 2.99 & -1.396 & 1.56 & 5.5344 & 2.75 & 0.3367 & 32.87 & 2.42 & 0.366 \\
\hline I.C & -17.34 & 7.09 & -1.047 & 2.44 & -1.544 & 1.37 & 6.6879 & 1.76 & 0.605 & 16.1 & 2.51 & 0.406 \\
\hline I.D & -18.79 & 4.32 & -0.699 & 2.68 & -1.426 & 1.03 & 5.2571 & 2.3 & 1.2377 & 7.6 & 2.02 & 0.427 \\
\hline I.E & -19.94 & 4.51 & -0.763 & 2.69 & -1.511 & 1.03 & 6.3336 & 1.85 & 0.3091 & 29.27 & 2.24 & 0.492 \\
\hline II.A1 & -22.98 & 4.48 & -0.989 & 2.12 & -1.564 & 1.17 & 6.3087 & 2.38 & 0.944 & 13.21 & 2.51 & 0.443 \\
\hline II.B1 & -22.22 & 3.82 & -0.477 & 3.01 & -1.505 & 0.94 & 5.7652 & 1.57 & 0.4317 & 21.8 & 1.9 & 0.56 \\
\hline II.C1 & -26.91 & 3.5 & -0.672 & 2.34 & -1.628 & 1.06 & 4.7481 & 2.23 & 0.7217 & 15.22 & 1.98 & 0.505 \\
\hline II.E1 & -13.75 & 9.47 & -0.756 & 4.19 & -1.361 & 1.52 & 7.2542 & 2.13 & 0.8637 & 12.46 & 2.42 & 0.46 \\
\hline II.B2 & -7.97 & 21.64 & -0.708 & 5.46 & -1.26 & 2.09 & 7.6083 & 2.03 & 1.0852 & 10.88 & 2.28 & 0.427 \\
\hline II.C2 & -30.94 & 2.86 & -0.769 & 2.01 & -1.702 & 0.94 & 5.6349 & 2.14 & 0.5568 & 20.79 & 2.34 & 0.539 \\
\hline II.D2 & -18.27 & 6.6 & -0.827 & 3.33 & -1.572 & 1.25 & 6.7571 & 1.85 & 0.7563 & 12.41 & 2.42 & 0.467 \\
\hline II.E2 & -19 & 5.49 & -0.622 & 3.42 & -1.444 & 1.19 & 6.0333 & 2.05 & 0.6112 & 16.97 & 2.05 & 0.501 \\
\hline II.F2 & -28.23 & 4.64 & -1.069 & 2.71 & -1.72 & 1.49 & 6.1214 & 2.88 & 0.4829 & 29.41 & 2.69 & 0.486 \\
\hline
\end{tabular}




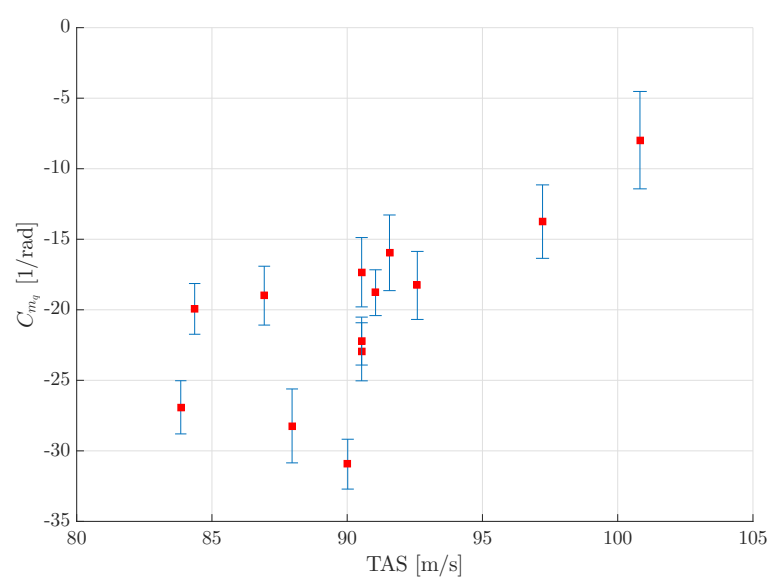

(a) Identified pitching moment due to $q$

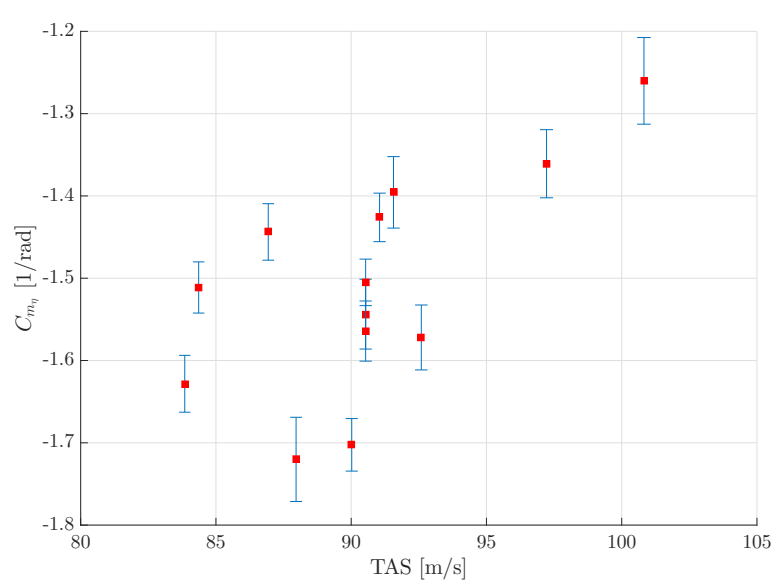

(c) Identified pitching moment due to $\eta$

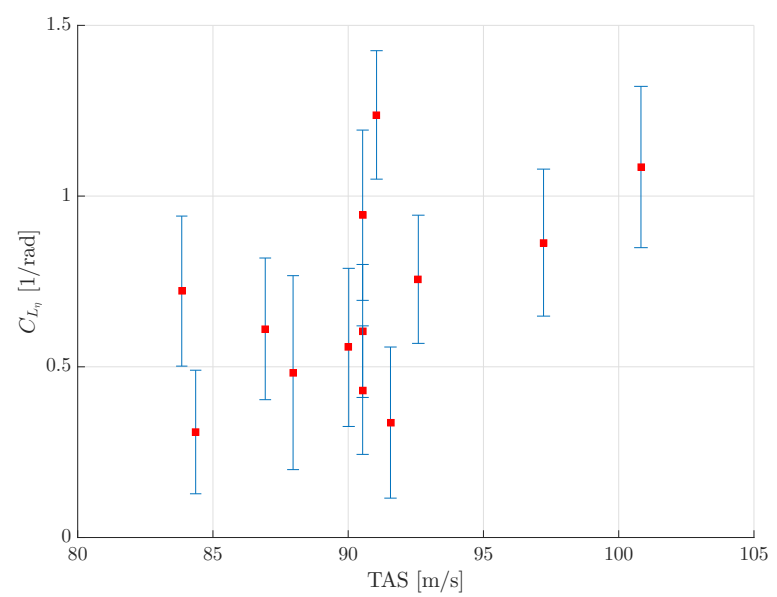

(e) Identified lift force due to $\eta$

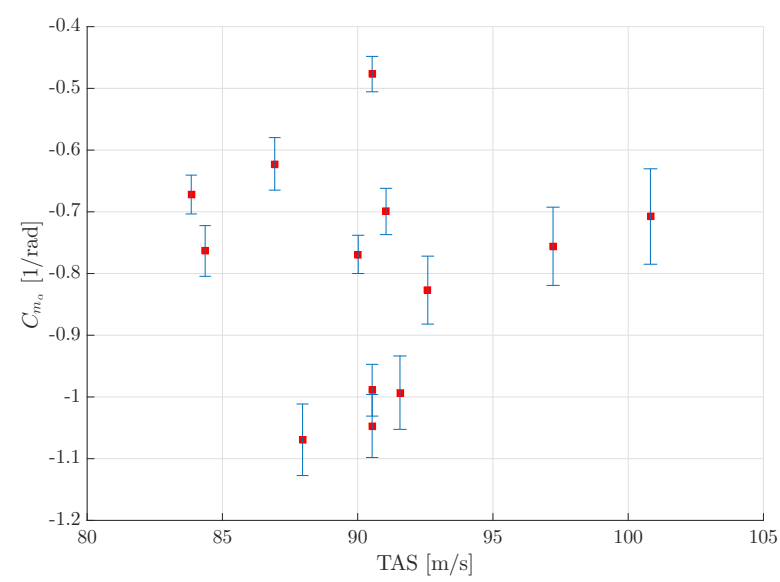

(b) Identified pitching moment due to $\alpha$

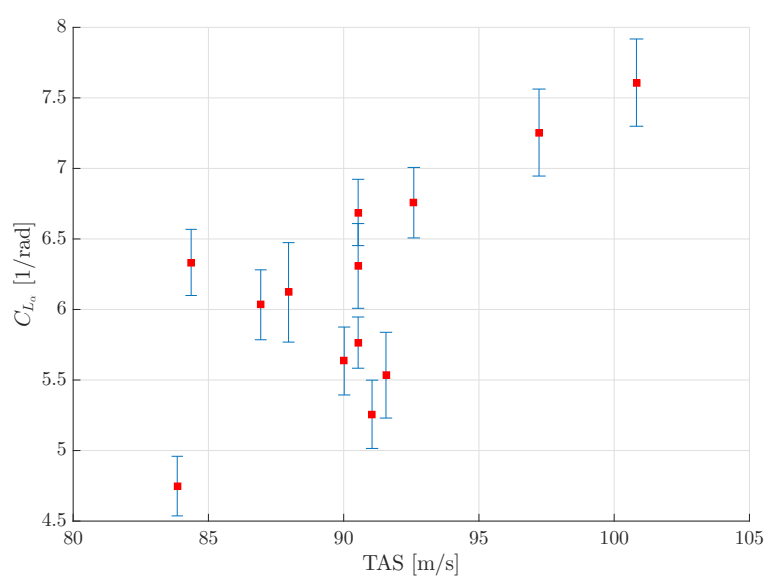

(d) Identified lift force due to $\alpha$

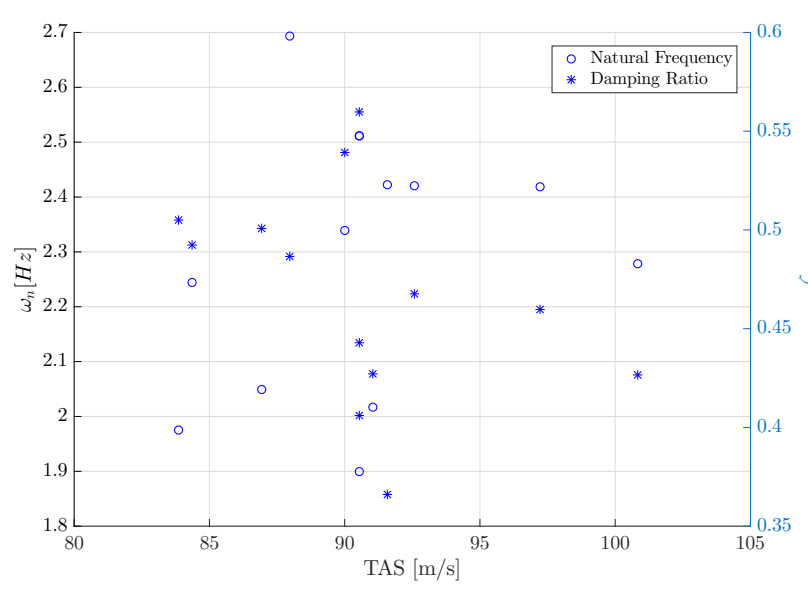

(f) Identified SPPO frequency and damping

Fig. 4. Parameter identification results 

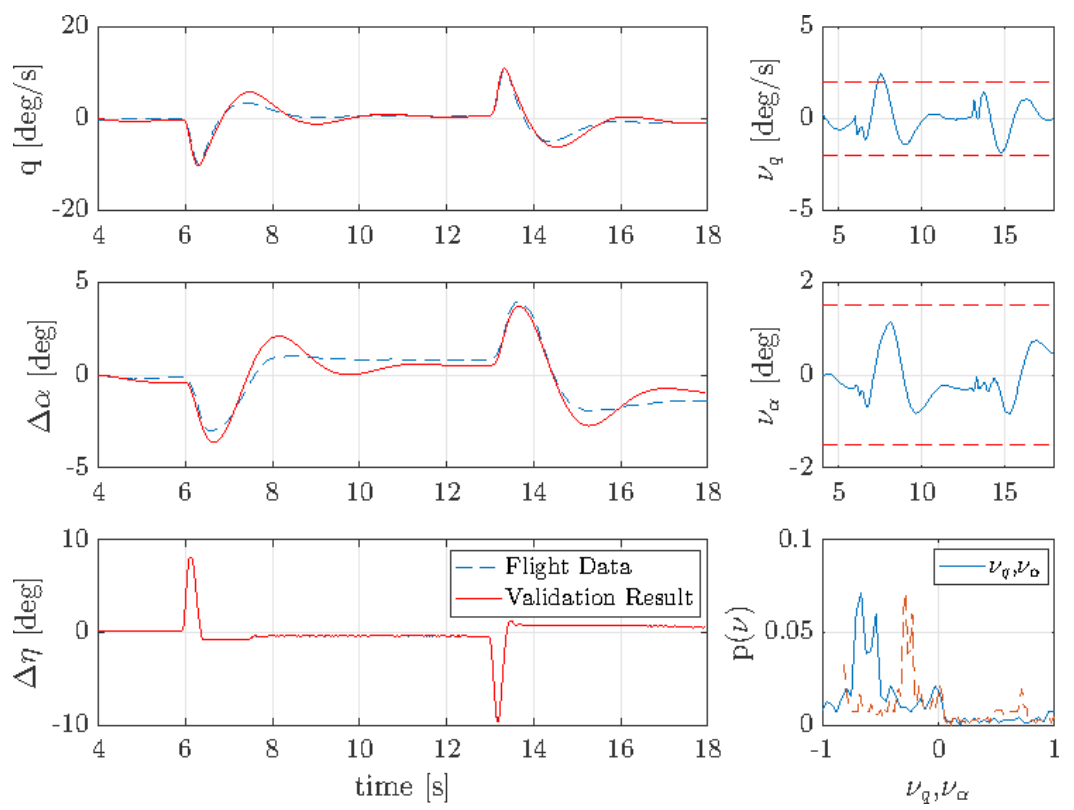

Fig. 5. Validation result 1: Flight I.B
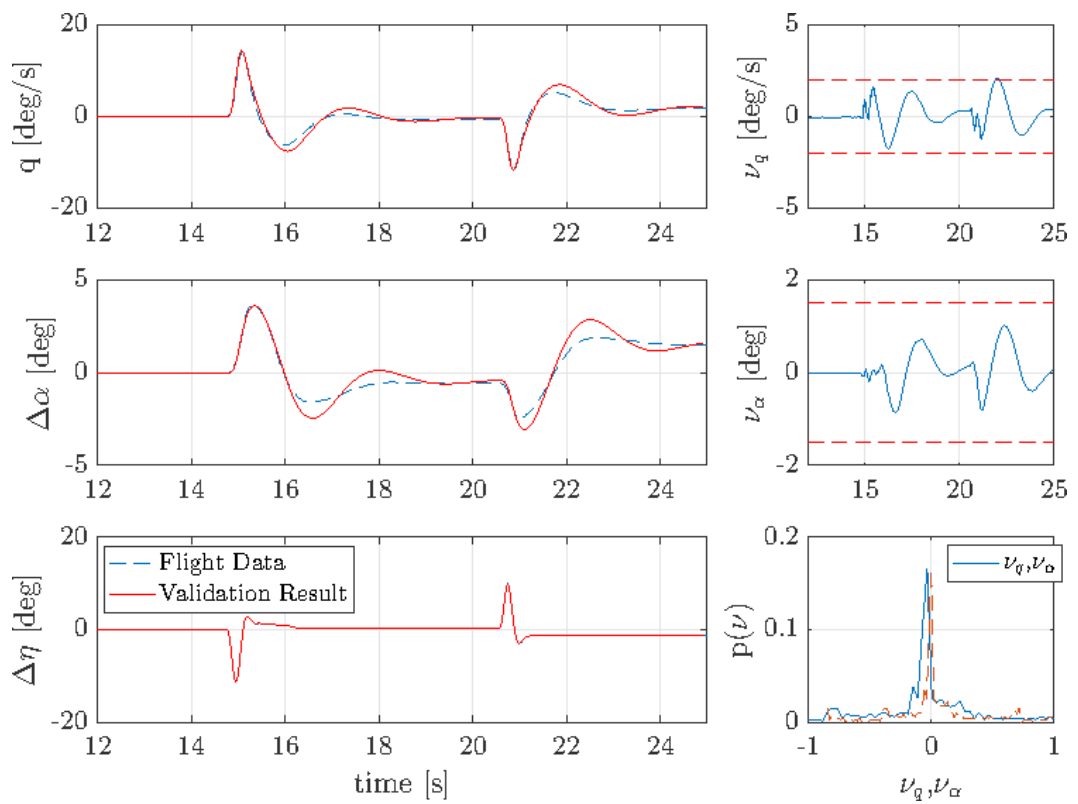

Fig. 6. Validation result 2: Flight II.A2 

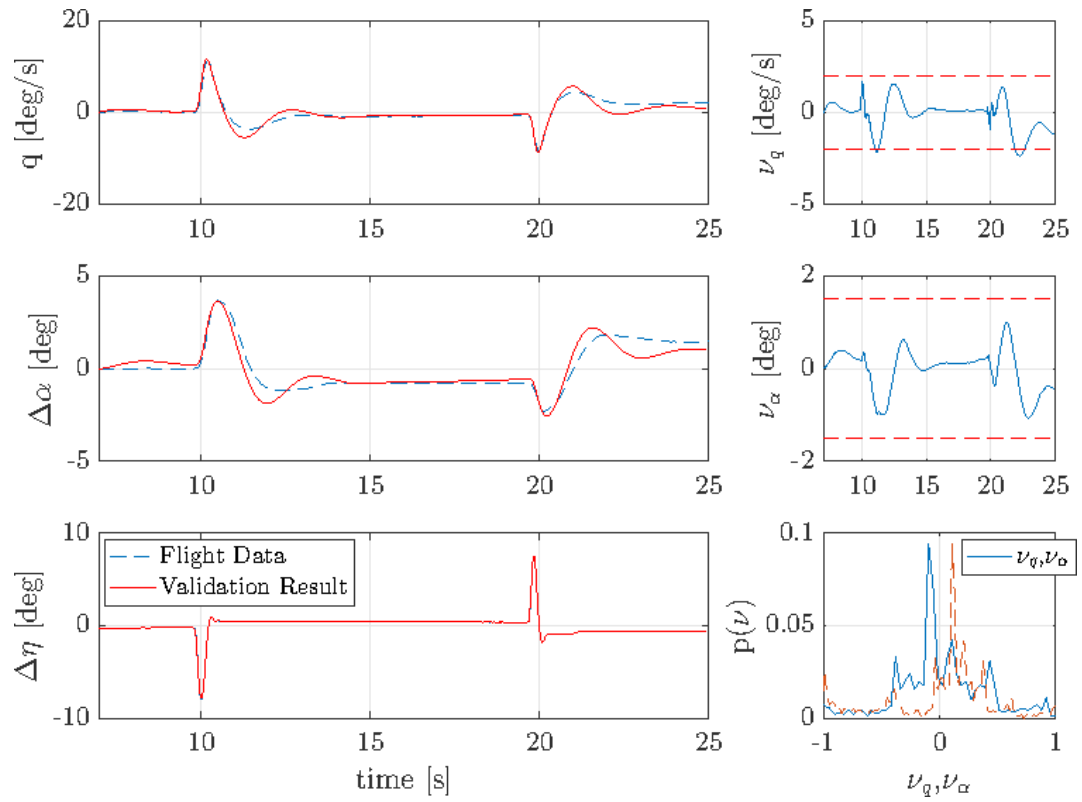

Fig. 7. Validation result 3: Flight II.D1

\section{Conclusions}

The Jetstream 31 G-NFLA aircraft is the workhorse of the NFLC that is used extensively for both research and teaching purposes. It has been the subject of much theoretical modelling that rely on empirical methods and CFD. There has also been a significant effort put towards experimental modelling in the wind tunnels. The focus of past work has been on characterising the aircraft from a static aerodynamics perspective, which has meant that flight dynamic parameters have not received much attention. The work discussed in this paper aims to fill this gap by analysing data from sixteen flight tests that allow: (a) another form of validation for past work (mainly through the estimation of derivatives such as $C_{L_{\alpha}}$ and $C_{m_{\alpha}}$ and, (b) the generation of stability and control derivatives such as $C_{m_{q}}$ and $C_{m_{\eta}}$. Here, the aircraft's short period pitch oscillation mode characteristics are identified using the least squares method and reduced order state-space model. The need for pre-processing regressors due to the effects of sensor location and instrumentation delays is highlighted. It has also been shown that time-shifting the regressors based on relative locations of the angle of attack vanes and the IMU results in significant reductions in uncertainty bounds of the estimated aeroderivatives and a model that provides a closer match to flight test data. The estimated models are validated using separate flight test data and the variations in aeroderivatives over a range of airspeeds and CG positions are also presented. 
2017-06-20

\section{Regressor time-shifting to identify}

longitudinal stability and control

derivatives of the Jetstream 3102

\section{Yusuf, Sezsy}

\section{Elsevier}

Yusuf S, Lone M, Cooke A, Lawson N. (2017) Regressor time-shifting to identify longitudinal stability and control derivatives of the Jetstream 3102. Aerospace Science and Technology, Volume 69, October 2017, pp. 218-225

http://dx.doi.org/10.1016/j.ast.2017.06.003

Downloaded from Cranfield Library Services E-Repository 